\title{
OFF-DIAGONAL LONG-RANGE ORDER, RESTRICTED GAUGE TRANSFORMATIONS, AND AHARONOV-BOHM EFFECT IN CONDUCTORS
}

\author{
Murray Peshkin* \\ Physics Division, Argonne National Laboratory, Argonne, IL 60439-4843
}

\begin{abstract}
The Hamiltonian describing a conductor surrounding an external magnetic field contains a nonvanishing vector potential in the volume accessible to the electrons and nuclei of which the conductor is made. That vector potential cannot be removed by a gauge transformation. Nevertheless, a macroscopic normal conductor can experience no Aharonov-Bohm effect. That is proved by assuming only that a normal conductor lacks off-diagonal long-range order (ODLRO). Then by restricting the Hilbert space to density matrices which lack ODLRO, it is possible to introduce a restricted gauge transformation that removes the interaction of the conductor with the vector potential.
\end{abstract}

PACS numbers: 03.65.Bz, $03.65-\mathrm{w}$ 
The question has sometimes been raised as to whether the Aharonov-Bohm (AB) effect [1.22 can be shielded by a conductor that surrounds the magnetic field, as in Fig. 1a. The beam particle induces charges and currents in the conductor. Those charges and currents may have their own $\mathrm{AB}$ effect as they encircle the magnetic flux, and that may compensate the $\mathrm{AB}$ effect on the beam particle.

There are also the more usual image charge and induced current effects, which have nothing to do with any interaction between the conductor and the external magnetic field. Image charges and induced currents act back on the beam particle and affect its motion. Those effects are not considered here. They are negligible in current experiments on the AB effect. In addition, they are at least quadratic in the charge of the beam particle, whereas the $\mathrm{AB}$ effect moves interference fringes proportionally to the charge of the beam particle for small fields. [The limiting case of diffraction by a flux line of vanishing width is exceptional because the zero-flux diffraction vanishes in that limit.]

Experimentally [2], we know that $\mathrm{AB}$ effect is observed at its full expected strength although the magnetic field is always surrounded by a conductor. However, the beam particle typically has a velocity above $10^{10} \mathrm{~cm} / \mathrm{sec}$ and the size of the scattering center is typically microns, so the frequencies to which the conductor would have to respond would be of order $10^{14} \mathrm{hz}$, approaching plasmon frequencies in metals, and one may speculate that shielding effects which may exist at lower frequencies would not have been seen in the experiments performed to date because the conductors could not react quickly enough to the fields created by the fast beam particles. Experiments with slower beam particles would perhaps have a better chance to exhibit shielding of the $\mathrm{AB}$ effect because there a close-coupling approximation, wherein the charge and current distributions in the conductor follow the beam particle adiabatically around the conductor, should apply. If such a phenomenon should exist for slower beam particles, it might raise the possibility of using $\mathrm{AB}$ effect to probe properties of a macroscopic shield in some way analogous to the very productive experiments now done with mesoscopic circuits.

The answer appears to be no; there can be no such shielding effect by a macroscopic 
conductor for beam particles of any energy. That answer was given by A.S. Goldhaber [3], both for normal and for superconducting conductors. For superconducting shields, the key point is the flux quantization. In the presence of a superconducting shield, the magnetic flux must be a multiple of $h c / 2 e$, half of Londons unit. However, the charge carriers have effectively charge $2 e$. Therefore the AB phase shift of the superconducting electrons, $(2 \pi) \times($ charge $) \times($ flux $)$, equals $2 \pi$ and gives rise to no observable effect.

For normal shields, Goldhaber's analysis relies upon specific and rather subtle dynamical properties of the conductor which may not be general. Here I give a proof that relies only on the most general property of normal matter, that it does not exhibit off-diagonal long-range order (ODLRO) [5]. The conduction electrons do not have a coherent phase around the ring and therefore cannot exhibit any $\mathrm{AB}$ effect of their own. In other words, the effects of the external flux on the dynamics of the conductor can be removed by a gauge transformation even though the vector potential cannot be removed by a gauge transformation. That statement has been made before [4] in a speculative way. Here I shall prove it.

To be gauge invariant, the Hamiltonian for the entire system must have the form

$$
H_{\mathbf{A}}=H\left(\mathbf{X}, \mathbf{P}-\frac{q}{c} \mathbf{A}(\mathbf{X}), \mathbf{S}, \mathbf{x}_{j}, \mathbf{p}_{j}-\frac{e_{j}}{c} \mathbf{A}\left(\mathbf{x}_{j}\right), \mathbf{s}_{j}\right)
$$

The vector potential A, assumed to be curl-free everywhere inside the conductor, is that due to the external magnetic field. Mutual magnetic interactions of the particles are to be expressed as functions of their dynamical variables. $\mathbf{X}, \mathbf{P}$, and $\mathbf{S}$ are the coordinate, canonical momentum, and spin of the beam particle. The $\mathbf{x}_{j}, \mathbf{p}_{j}$, and $\mathbf{s}_{j}$ are the coordinates, canonical momenta, and spins of all particles in the shield, electrons and nuclei. For an electron, the charge $q$ or $e_{j}$ is negative.

The vector potential cannot be removed by a gauge transformation, except for special values of the magnetic flux $\Phi$, because it must obey

$$
\oint \mathbf{A} \cdot d \mathbf{r}=\Phi
$$

The exceptional cases are those for which the flux obeys 


$$
\Phi=n \frac{h c}{e}
$$

with integer $n$.

If the conductor is simply connected, as in Fig. 1b, the interaction between the magnetic flux and the particles in the conductor can be removed from the Hamiltonian by a gauge transformation $U$ in the standard way. Within the domain of the Hamiltonian, i.e. when the coordinates $\mathbf{x}_{j}$ lie within the split-ring conductor of Fig. 1b,

$$
\begin{aligned}
\Psi^{\prime}\left(\mathbf{X}, \xi, \mathbf{x}_{j}, \xi_{j}, t\right) & =\bar{U} \Psi\left(\mathbf{X}, \xi, \mathbf{x}_{j}, \xi_{j}, t\right) \\
\bar{U} & =\prod_{j} U\left(\mathbf{x}_{j}\right) \\
U\left(\mathbf{x}_{j}\right) & =\exp \left\{\frac{i e_{j}}{\hbar c} \int^{\mathbf{x}_{j}} \mathbf{A}(\mathbf{r}) \cdot d \mathbf{r}\right\}
\end{aligned}
$$

where $\xi$ and $\xi_{j}$ are the values of $S_{z}$ and $s_{j z}$.

$$
\bar{H}_{\mathbf{A}}=\bar{U} H_{\mathbf{A}} \bar{U}^{-1}=H\left(\mathbf{X}, \mathbf{P}-\frac{q}{c} \mathbf{A}(\mathbf{X}), \mathbf{S}, \mathbf{x}_{j}, \mathbf{p}_{j}, \mathbf{s}_{j}\right)
$$

The interaction between the external field and the beam particle is retained in Eq. (5) through $\mathbf{A}(\mathbf{X})$.

The density operator $\rho$, which, along with $H$, determines the dynamics, obeys

$$
\bar{\rho}=\bar{U} \rho \bar{U}^{-1}
$$

Equivalently, the density matrix obeyst

$$
\begin{gathered}
\left\langle\mathbf{X}, \xi, \mathbf{x}_{1}, \xi_{1}, \cdots \mathbf{x}_{N}, \xi_{N}|\bar{\rho}(t)| \mathbf{X}^{\prime}, \xi^{\prime}, \mathbf{x}_{1}^{\prime} \xi_{1}^{\prime}, \cdots \mathbf{x}_{N}^{\prime}, \xi_{N}^{\prime}\right\rangle= \\
\bar{V}\left\langle\mathbf{X}, \xi, \mathbf{x}_{1}, \xi_{1}, \cdots \mathbf{x}_{N}, \xi_{N}|\rho(t)| \mathbf{X}^{\prime}, \xi^{\prime}, \mathbf{x}_{1}^{\prime} \xi_{1}^{\prime}, \cdots \mathbf{x}_{N}^{\prime}, \xi_{N}^{\prime}\right\rangle \\
\bar{V}=\prod_{j} V\left(\mathbf{x}_{j}, \mathbf{x}_{j}^{\prime}\right)=\prod_{j} \exp \left\{\frac{i e_{j}}{\hbar c} \int_{\mathbf{x}_{j}}^{\mathbf{x}_{j}^{\prime}} \mathbf{A}(\mathbf{r}) \cdot d \mathbf{r}\right\}
\end{gathered}
$$

\footnotetext{
${ }^{1}$ Following Ref. [5], the particles are in effect numbered and the statistics are imposed through the symmetry of the density matrix. For instance, if particles 1 and 2 are both electrons, then $\rho$ changes sign under $\left(\mathbf{x}_{1}, \xi_{1}\right) \Leftrightarrow\left(\mathbf{x}_{2}, \xi_{2}\right)$ and the same is true of the primed variables.
} 
For a simply-connected conductor, Eqs. (5) and (6) suffice to show that the action of the external magnetic field on the particles in the conductor is removed by a gauge transformation and therefore the external field has no physical effect. For a multiply-connected conductor such as the one in Fig. 1a, that proof fails because the unitary operator $U$ does not exist except for values of the magnetic flux that obey Eq. (3). For all other values of the flux, the function $U\left(\mathbf{x}_{j}\right)$ is multiple valued and it cannot carry a wave function within the domain of $H$ into a second wave function within the domain of $H$. Similarly, $V\left(\mathbf{x}_{j}, \mathbf{x}_{j}^{\prime}\right)$ is multiple-valued and cannot carry an acceptable density matrix into a second acceptable density matrix. The multiple valuedness can be removed by making a mathematical cut, for instance at the azimuthal angle $\phi=0$, so that the line integrals of $\mathbf{A}$ become single valued, but then the wave functions become discontinuous and the domain problem does not go away.

However, for a macroscopic normal conductor, the proof can be rescued by restricting the space of the density matrices to those which do not have ODLRO. Strictly, such density matrices obey

$$
\lim _{\left|\mathbf{x}_{j}-\mathbf{x}_{j}^{\prime}\right| \rightarrow \infty}\left\langle\mathbf{X}, \xi, \mathbf{x}_{1}, \xi_{1}, \cdots \mathbf{x}_{N}, \xi_{N}|\rho| \mathbf{X}^{\prime}, \xi^{\prime}, \mathbf{x}_{1}^{\prime} \xi_{1}^{\prime}, \cdots \mathbf{x}_{N}^{\prime}, \xi_{N}^{\prime}\right\rangle=0
$$

for each $j$ individually. I will take a macroscopic normal ring to be one for which

$$
\begin{array}{r}
\left\langle\mathbf{X}, \xi, \mathbf{x}_{1}, \xi_{1}, \cdots \mathbf{x}_{N}, \xi_{N}|\rho| \mathbf{X}^{\prime}, \xi^{\prime}, \mathbf{x}_{1}^{\prime} \xi_{1}^{\prime}, \cdots \mathbf{x}_{N}, \xi_{N}^{\prime}\right\rangle=0 \\
\text { when }\left|\mathbf{x}_{j}-\mathbf{x}_{j}^{\prime}\right|>a \text { for any } j
\end{array}
$$

where $a$ is some length less than half the length of the shortest path through the conducting ring that encircles the magnetic flux.

Now each $\int_{\mathbf{x}_{j}}^{\mathbf{x}_{j}^{\prime}} \mathbf{A}(\mathbf{r}) \cdot d \mathbf{r}$ in Eq. (4) can be made single-valued by requiring the integration path to obey

$$
\left|\mathbf{r}-\mathbf{x}_{j}\right|<a \quad \text { and } \quad\left|\mathbf{r}-\mathbf{x}_{j}^{\prime}\right|<a
$$

for every pair $\left(\mathbf{x}_{j}, \mathbf{x}_{j}^{\prime}\right)$ which obeys $\left|\mathbf{x}_{j}-\mathbf{x}_{j}^{\prime}\right|<a$. It is unnecessary to define $V$ for other pairs, because the density matrix in Eq. (7) vanishes for all those pairs. Equations (7) and 
(8) define a single-valued density matrix $\bar{\rho}$ which is gauge equivalent to $\rho$. There is no discontinuity problem because $\rho$ vanishes in the regions where $V$ has a jump in phase.

The same trick can be played on the Hamiltonian $H$. The gauge transformation

$$
\bar{H}=\bar{U} H \bar{U}^{-1}
$$

does not exist in general because it creates a multiple-valued Hamiltonian that has no meaning, but in the truncated space of density matrices that do not have ODLRO, that does not matter. The matrix elements of $\bar{H}$ can be defined by the restricted gauge transformation

$$
\begin{aligned}
& \left\langle\mathbf{X}, \xi, \mathbf{x}_{1}, \xi_{1}, \cdots \mathbf{x}_{N}, \xi_{N}|\bar{H}| \mathbf{X}^{\prime}, \mathbf{x}_{1}^{\prime} \xi_{1}^{\prime}, \cdots \mathbf{x}_{N}^{\prime}, \xi_{N}^{\prime}\right\rangle= \\
& \quad \bar{V}\left\langle\mathbf{X}, \xi, \mathbf{x}_{1}, \xi_{1}, \cdots \mathbf{x}_{N}, \xi_{N}|H| \mathbf{X}^{\prime}, \xi^{\prime}, \mathbf{x}_{1}^{\prime}, \xi_{1}^{\prime}, \cdots \mathbf{x}_{N}^{\prime}, \xi_{N}^{\prime}\right\rangle
\end{aligned}
$$

whenever all pairs $\left(\mathbf{x}_{j}, \mathbf{x}_{j}^{\prime}\right)$ obey $\left|\mathbf{x}_{j}-\mathbf{x}_{j}^{\prime}\right|<a$. Other matrix elements of $\bar{H}$ can be taken to vanish because they only multiply vanishing matrix elements of the density matrix. The multiple-valuedness problem has been eliminated and once again the interaction of the external magnetic field with the particles in the conductor has been removed from the Hamiltonian and the density matrix.

The assumption that the density matrix exhibits no off-diagonal long-range order at any time implies the assumption that the Schroedinger equation

$$
i \hbar \frac{\partial \rho}{\partial t}=[H, \rho]
$$

preserves the absence of ODLRO. This proof would therefore not apply to the unlikely situation where the passage of the beam particle somehow jostles the conductor into a superconducting state.

For mesoscopic circuits, on the nanometers scale, this proof fails because the dimensions of the circuits are smaller than the length $a$ which measures the range of the off-diagonal order. Finding the circuit size beyond which measured AB effects in the conductor disappears might give a direct, albeit only semi-quantitative, measure of $a$. 


\section{ACKNOWLEDGMENT}

I thank F. Coester for useful discussions. This work is supported by the U.S. Department of Energy, Nuclear Physics Division, under contract W-31-109-ENG-38. 


\section{REFERENCES}

* Electronic address: peshkin@anl.gov

[1] Y. Aharonov and D. Bohm, Phys. Rev. 115, 485 (1959).

[2] M. Peshkin and A. Tonomura, The Aharonov-Bohm Effect, Lecture Notes in Physics, Vol. 340 (Springer-Verlag, Berlin 1989).

[3] A. S. Goldhaber, in Proceedings of the Santa Fe Workshop "Foundations of Quantum Mechanics", eds. T. D. Black et al. (World Scientific, Singapore 1992) p. 221.

[4] M. Peshkin, in Proceedings of the International Symposium "Foundations of Quantum Mechanics in the Light of New Technology, eds. S. Kamefuchi et al. (Physical Society of Japan, Tokyo 1984) p. 56.

[5] C. N. Yang, Rev. Mod. Phys. 34, 694 (1962). 


\section{FIGURES}

FIG. 1. A conductor (shaded) surrounding a magnetic field region (black). a) Intact, multiply connected, ring. b) Split, simply connected, ring. 


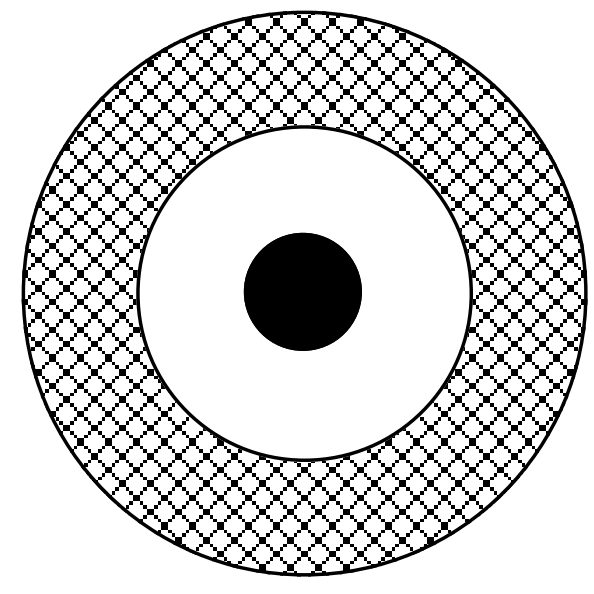

(a)

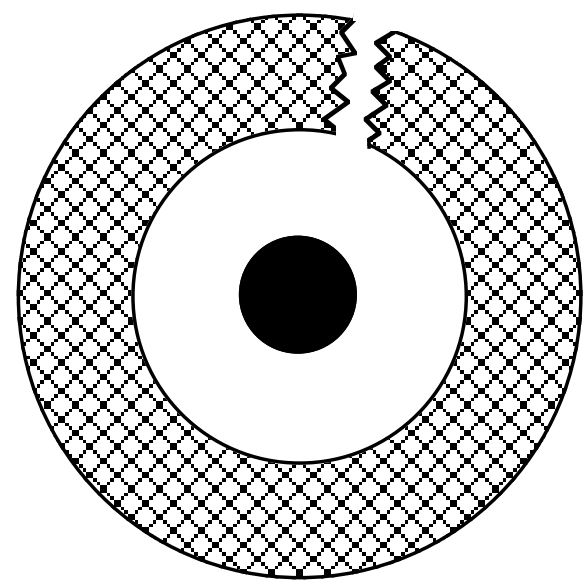

(b)

Fig. 1. A conductor (shaded) surrounding a magnetic field region (black).

(a) Intact, multiply connected, ring.

(b) Split, simply connected, ring. 